\title{
The Finite Element Numerical Modelling of 3D Magnetotelluric
}

\author{
Ligang $\mathrm{Cao}^{1}$ and Yun Liu' \\ ${ }^{1}$ Key Laboratory of Earth Exploration and Information Techniques, Chengdu University of Technology, Ministry of Education, \\ Chengdu 610059, China \\ ${ }^{2}$ Institute of Geochemistry Chinese Academy of Sciences, Guiyang 550002, China \\ Correspondence should be addressed to Ligang Cao; caoligang08@cdut.cn
}

Received 24 March 2014; Revised 18 June 2014; Accepted 15 July 2014; Published 6 August 2014

Academic Editor: Davide Spinello

Copyright (C) 2014 L. Cao and Y. Liu. This is an open access article distributed under the Creative Commons Attribution License, which permits unrestricted use, distribution, and reproduction in any medium, provided the original work is properly cited.

The ideal numerical simulation of 3D magnetotelluric was restricted by the methodology complexity and the time-consuming calculation. Boundary values, the variation of weighted residual equation, and the hexahedral mesh generation method of finite element are three major causes. A finite element method for 3D magnetotelluric numerical modeling is presented in this paper as a solution for the problem mentioned above. In this algorithm, a hexahedral element coefficient matrix for magnetoelluric finite method is developed, which solves large-scale equations using preconditioned conjugate gradient of the first-type boundary conditions. This algorithm is verified using the homogeneous model, and the positive landform model, as well as the low resistance anomaly model.

\section{Introduction}

Both 1D and 2D MT numerical simulation technologies have brought benefits to economy with their maturity. However, they are both the approximate processing of a 3D numerical simulation, which is the closest to the real geological body model [1-9].

With the development in computer science and computing technology, numerical simulation of 3D MT data has become the research focus. Shizhe [10] proposed the conjecture and theoretical basis of "Hexahedral mesh generation method for finite element" and "Boundary element method in 3D numerical simulation" [11]. However, there are only a few publications regarding successful scientific research on 3D numerical simulations [12-15]. The major difficulties lie in three aspects: (1) insufficient computing capability due to limited CPU memory; (2) immature parallel algorithm on multicore CPU's; (3) limited boundary element 3D MT numerical simulation on pure terrain uniform earth. Shu and Chen [16] proposed a 3D forward method in horizontal electric dipole under the magnetotelluric response by using a vector finite element method. After combining scalar finite elements and vector finite elements together,
Mitsuhata and Uchida [17] conducted forward computation and error analysis for the two 3D COMMEMI models using $T \_\Omega$ formula, which verified the correctness of the program as well. Under the conditions of tetrahedral elements, Linping and Shikun [18] derived the analytical expression for the underground varying conductivity to calculate the threedimensional magnetotelluric finite element equation. Nam et al. [19] proposed the 3D magnetotelluric response simulation based on hexahedral-vector finite element method and simulated the 3D terrain using isoparametric element method. As for the underground complex geological body, only a few papers are available from public sources [20-27].

This paper deduces the hexahedral element coefficient matrix of the magnetotelluric finite element method based on Xu Shizhe's work. It uses preconditioned conjugate gradient method to solve large-scale equations under the firsttype boundary conditions. This algorithm can significantly improve the computation efficiency. Via computing analysis of homogeneous model, undulating terrain model, and 3D model, the 3D low resistivity abnormal morphology can be clearly reflected. As the frequency decreases, the errors between the calculation results and analytical solutions are found gradually decreasing. 


\section{The Boundary Problem of 3D MT}

A 3D geology space model is shown in Figure 1. The space is divided into two layers, air and underground, by an undulating $\left(\mathrm{A}^{\prime} \mathrm{B}^{\prime} \mathrm{C}^{\prime} \mathrm{D}^{\prime}\right)$. The surface of $\mathrm{ABCD}$ is the infinite boundary of upper air which is about $100 \mathrm{~km}$ height, and the surface of EFGH is infinite boundary of underground which is about $30 \mathrm{~km}$ beneath ground. In addition, there are four infinite vertical boundary surfaces: ABFE, BFGC, DCGH and ADHE, all of which are about $30 \mathrm{~km}$ away from the center area of measurement.

The source of natural alternating electromagnetic field is located on the surface of ABCD. When the electromagnetic wave passes through the atmosphere and then enters the underground, the whole 3D geology space becomes the study field of MT method due to continuous electric field on the tangential direction and magnetic fields on the normal direction.

2.1. The Boundary Value Problem of MT Method. The electromagnetic waves from the magnetotelluric field source vertically incident into the underground as harmonic plane waves with a time harmonic factor $e^{-i \omega t}$. Maxwell coupled magnetotelluric field equations in frequency domain as

$$
\begin{gathered}
\nabla \times E=i \omega \mu H, \\
\nabla \times H=(\sigma-i \omega \varepsilon) E,
\end{gathered}
$$

where $E$ is the electric vector, $H$ is the magnetic vector, $\omega$ is angular frequency, $\mu$ is the medium permeability when the permeability of air is $1.2567 \times 10^{-6} \mathrm{H} / \mathrm{m}, \sigma$ is the medium conductivity or the reciprocal of medium resistivity, $\varepsilon$ is the dielectric medium constant, and the dielectric constant of air is $8.85 \times 10^{-12} \mathrm{~F} / \mathrm{m}$.

Curling both sides of the electric equations (1), the differential electric equation can be obtained as follows:

$$
\begin{aligned}
& \nabla \times(\nabla \times E)-k^{2} E=0, \\
& \nabla \times(\nabla \times H)-k^{2} H=0,
\end{aligned}
$$

where

$$
k^{2}=i \omega \mu \sigma+\omega^{2} \varepsilon \mu
$$

The equations of electric and magnetic have the same form that can be written in one unification equation. Substituting $E$ and $H$ for $U$ yields

$$
\nabla \times(\nabla \times U)-k^{2} U=0 .
$$

The vector $U$ can be rewritten to the vector form with three components:

$$
U=U_{x} e_{x}+U_{y} e_{y}+U_{z} e_{z}
$$

where, $e_{x}, e_{y}$, and $e_{z}$ are the unit vectors in the directions of $x, y$, and $z$.

\subsubsection{When It Is Working in TE Model}

(a) In the surface of ABCD shown in Figure 1, the field source is the electric vector $E_{x}$. As the ratio of electric and magnetic, $U_{x}$ has no impact to the final result. Therefore, $U_{x}$ can be either zero or nonzero value. Here $U_{x}=1 . U_{y}$ and $U_{z}$ are all zero as well since there are no components of $U$ in $x$ and $y$ directions.

(b) In the four vertical boundaries shown in Figure 1, the propagation direction of EM wave in magnetic field is vertical downward, being perpendicular to normal direction of the boundary, which is $E \times H \perp \Gamma$. Therefore, $\partial U / \partial n$ equals zero.

(c) In the surface of EFGH in Figure 1, the underground can be assumed as a homogenous medium with a consideration that this surface is beyond the study area. The electromagnetic field attenuates in a normal way, which follows the index law during downward propagation. The vector $U$ has no value in directions $y$ and $z$.

$$
U_{x}=c e^{-\chi z}, \quad U_{y}=0, \quad U_{z}=0
$$

where $c$ is a constant factor with any nonzero value, $\chi=$ $\sqrt{-i \omega \mu \sigma}$, and $\sigma$ is the homogenous conductivity below the infinite surface EFGH. Derivative of (6) is obtained as follows:

$$
\frac{\partial U_{x}}{\partial n}=\frac{\partial U_{x}}{\partial z}=-\chi U_{x}
$$

2.1.2. When It Is Working in TM Model. The field source is one component of the magnetic vector $H_{x}$. When magnetic field passes through the atmosphere, attenuation barely happens. Therefore, the boundary conditions of $\mathrm{ABCD}$ can be replaced by $\mathrm{A}^{\prime} \mathrm{B}^{\prime} \mathrm{C}^{\prime} \mathrm{D}^{\prime} \mathrm{s}$ in the TE model.

\section{The Variation Problem of Weighted Residual Equation}

Both sides of (4) are multiplied by $\delta U$ and integrated on the whole region, showing the result as follows:

$$
\int_{\Omega}\left[\nabla \times(\nabla \times U)-k^{2} U\right] \cdot \delta U d \Omega=0
$$

where

$$
\delta U=\delta U_{x} e_{x}+\delta U_{y} e_{y}+\delta U_{z} e_{z} .
$$

According to the vector identities

$$
(\nabla \times A) \cdot B=\nabla \cdot(A \times B)+(\nabla \times B) \cdot A .
$$

Equation (8) will be

$$
\begin{aligned}
\int_{\Omega} \nabla \times(\nabla \times U) \cdot \delta U d \Omega= & \int_{\Omega} \nabla \cdot[(\nabla \times U) \times \delta U] d \Omega \\
& +\int_{\Omega} \nabla \times \delta U \cdot \nabla \times U d \Omega .
\end{aligned}
$$




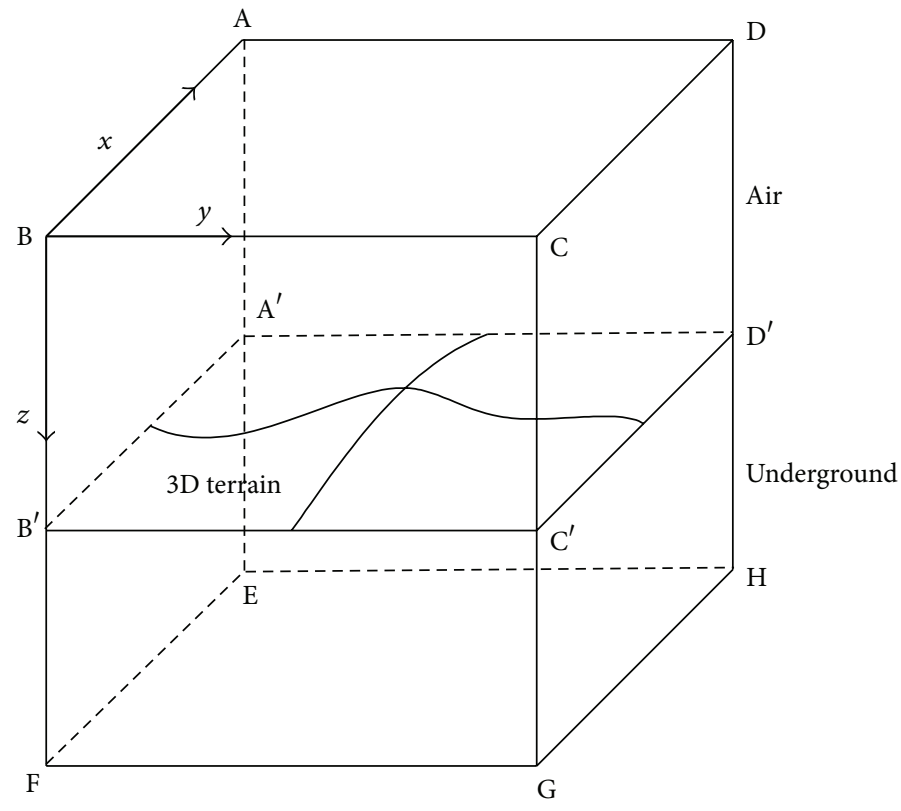

FIgURE 1: 3D space of the study.

In which,

$$
\int_{\Omega} \nabla \cdot[(\nabla \times U) \times \delta U] d \Omega=\int_{\Gamma}(\nabla \times U) \times \delta U \cdot d \Gamma .
$$

According to the boundary condition

(a) in the surfaces $\mathrm{ABCD}$ and $\mathrm{A}^{\prime} \mathrm{B}^{\prime} \mathrm{C}^{\prime} \mathrm{D}^{\prime}, \delta U=0$ due to fixed $U$ in these surfaces;

(b) in the four infinite vertical surfaces, $(\nabla \times U) \times \delta U \cdot d \Gamma=$ 0 , because the reflection of the anomalous field can be ignored. Then the electric field $U$ and $\delta U$ have one component $x$ only;

(c) in the surface EFGH, $(\nabla \times U) \times \delta U=-\left(\partial U_{x} / \partial z\right) \delta U_{x} e_{z}$. Substituting all the boundary conditions in (12) yields

$$
\begin{gathered}
\int_{\Omega} \nabla \times \delta U \cdot \nabla \times U d \Omega-\int_{\Omega} k^{2} U \cdot \delta U d \Omega \\
+\int_{\mathrm{EFGH}} \chi U_{x} \delta U_{x} d \Gamma=0 .
\end{gathered}
$$

\section{Hexahedral Mesh Generation}

Hexahedral element mesh is used to make Figure 1 into a $3 \mathrm{D}$ geology space as shown in Figure 2. Obviously, the entire space of 3D geology is meshed into the amount of hexahedral elements.

The vertices of each hexahedral element are sorted as shown in Figure 3.

\section{Unit Analysis}

Using the finite element method to solve differential equations requires a mesh representation of the target area, such

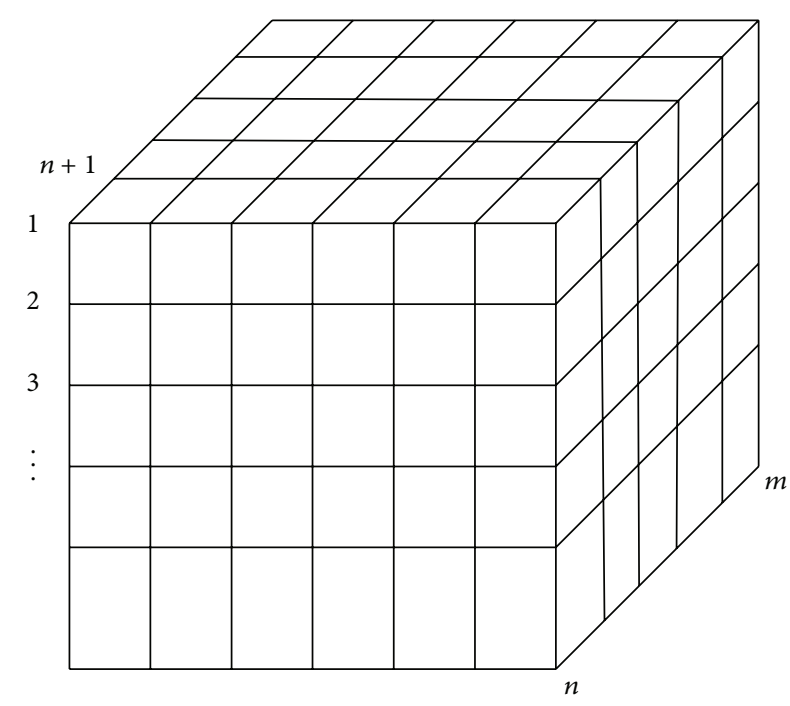

FIGURE 2: Hexahedral mesh.

as the widely used hexahedral mesh. Figure 4(a) is the parent element of the hexahedron and Figure 4(b) is the subelement. The coordinate correspondences between them are

$$
x=x_{0}+\frac{a}{2} \xi, \quad y=y_{0}+\frac{b}{2} \eta, \quad z=z_{0}+\frac{c}{2} \zeta,
$$

where $x_{0}, y_{0}$, and $z_{0}$ is the center coordinate of sub element; $a, b, c$ is the lengths of three edges. The differential relations between them are

$$
d x=\frac{a}{2} d \xi, \quad d y=\frac{b}{2} d \eta, \quad d z=\frac{c}{2} d \zeta .
$$




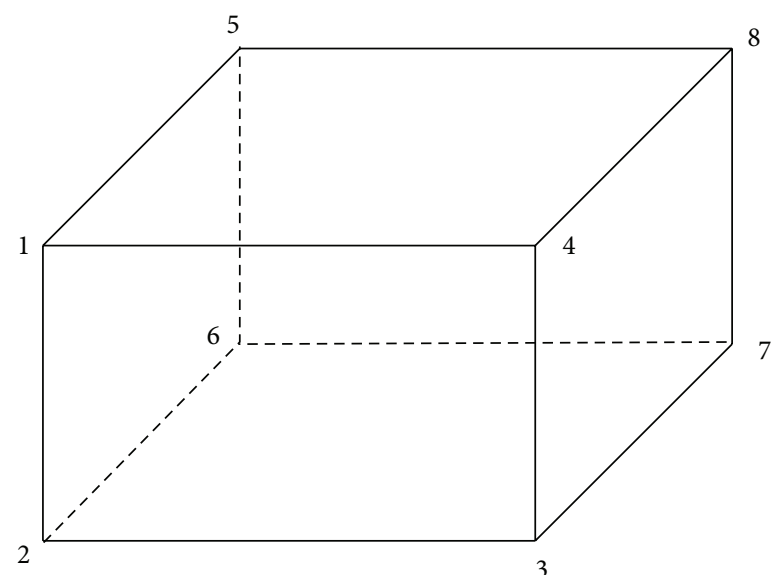

FIGURE 3: The vertices of hexahedral mesh.

The shape function is defined as

$$
N_{i}=\frac{1}{8}\left(1+\xi_{i} \xi\right)\left(1+\eta_{i} \eta\right)\left(1+\zeta_{i} \zeta\right)
$$

where $\xi_{i}, \eta_{i}, \zeta_{i}$ are the coordinates of vertex $i$ in the parent unit.

The interpolation function is

$$
E_{x}=N^{T} E_{x}, \quad E_{y}=N^{T} E_{y}, \quad E_{z}=N^{T} E_{z},
$$

where $N^{T}=\left(N_{1}, \ldots, N_{8}\right), E_{x}{ }^{T}=\left(E_{x 1}, \ldots, E_{x 8}\right), E_{y}{ }^{T}=$ $\left(E_{y 1}, \ldots, E_{y 8}\right), E_{z}^{T}=\left(E_{z 1}, \ldots, E_{z 8}\right)$. Therefore

$$
\begin{gathered}
E=N^{T}\left(E_{x} e_{x}+E_{y} e_{y}+E_{z} e_{z}\right) \\
\delta E=N^{T}\left(\delta E_{x} e_{x}+\delta E_{y} e_{y}+\delta E_{z} e_{z}\right),
\end{gathered}
$$

where $\delta E_{x}, \delta E_{y}$, and $\delta E_{z}$ are the variations of $E_{x}, E_{y}$, and $E_{z}$.

\section{The Form of Unit Coefficient Matrix}

The regional integral of (13) can be decomposed into the integral of each unit:

$$
\begin{aligned}
& \sum_{\Omega} \int_{e} \nabla \times \delta E \cdot \nabla \times E d \Omega-\sum_{\Omega} \int_{\Omega} k^{2} E \cdot \delta E d \Omega \\
& +\sum_{\mathrm{EFGH}} \int_{1234} \chi E_{x} \delta E_{x} d \Gamma=0 .
\end{aligned}
$$

In one unit $e$

$$
\begin{gathered}
\nabla \times E=E_{e}^{T}\left(\alpha e_{x}+\beta e_{y}+\gamma e_{z}\right), \\
\nabla \times \delta E=\delta E_{e}^{T}\left(\alpha e_{x}+\beta e_{y}+\gamma e_{z}\right),
\end{gathered}
$$

where

$$
\begin{gathered}
E_{e}{ }^{T}=\left(E_{x}{ }^{T}, E_{y}{ }^{T}, E_{z}{ }^{T}\right), \quad \delta E_{e}{ }^{T}=\left(\delta E_{x}{ }^{T}, \delta E_{y}{ }^{T}, \delta E_{z}{ }^{T}\right) \\
\alpha^{T}=\left[0,-\left(\frac{\partial N}{\partial z}\right)^{T},\left(\frac{\partial N}{\partial y}\right)^{T}\right], \\
\beta^{T}=\left[-\left(\frac{\partial N}{\partial z}\right)^{T}, 0,\left(\frac{\partial N}{\partial x}\right)^{T}\right], \\
\gamma^{T}=\left[-\left(\frac{\partial N}{\partial y}\right)^{T},\left(\frac{\partial N}{\partial x}\right)^{T}, 0\right], \\
\left(\frac{\partial N}{\partial x}\right)^{T}=\left(\frac{\partial N_{1}}{\partial x}, \ldots, \frac{\partial N_{8}}{\partial x}\right) \quad \frac{\partial N_{i}}{\partial x}=\frac{\partial N_{i}}{\partial \xi} \frac{2}{a}, \\
\left.\left(\frac{\partial N}{\partial z}\right)^{T}=\left(\frac{\partial N_{1}}{\partial y}, \ldots, \frac{\partial N_{8}}{\partial y}\right) \quad \frac{\partial N_{i}}{\partial y}=\frac{\partial N_{i}}{\partial \eta} \frac{2}{b}, \ldots, \frac{\partial N_{8}}{\partial z}\right) \quad \frac{\partial N_{i}}{\partial z}=\frac{\partial N_{i}}{\partial \zeta} \frac{2}{c} \\
i=1, \ldots, 8 .
\end{gathered}
$$

Substituting (20) into the first integral of (19)

$$
\int_{e} \nabla \times \delta E \cdot \nabla \times E d \Omega=\delta E_{e}^{T} K_{1 e} E_{e}
$$

where

$$
K_{1 e}=\int_{e}\left(\alpha \alpha^{T}+\beta \beta^{T}+\gamma \gamma^{T}\right) d \Omega .
$$

Its matrix form is

$$
\begin{aligned}
K_{1 e} & =\left[\begin{array}{lll}
K 11 & K 12 & K 13 \\
K 21 & K 22 & K 23 \\
K 31 & K 32 & K 33
\end{array}\right], \\
K 11 & =\int_{e}\left[\left(\frac{\partial N}{\partial y}\right)\left(\frac{\partial N}{\partial y}\right)^{T}+\left(\frac{\partial N}{\partial z}\right)\left(\frac{\partial N}{\partial z}\right)^{T}\right] d \Omega, \\
K 22 & =\int_{e}\left[\left(\frac{\partial N}{\partial x}\right)\left(\frac{\partial N}{\partial x}\right)^{T}+\left(\frac{\partial N}{\partial z}\right)\left(\frac{\partial N}{\partial z}\right)^{T}\right] d \Omega, \\
K 33 & =\int_{e}\left[\left(\frac{\partial N}{\partial x}\right)\left(\frac{\partial N}{\partial x}\right)^{T}+\left(\frac{\partial N}{\partial y}\right)\left(\frac{\partial N}{\partial y}\right)^{T}\right] d \Omega, \\
K 21 & =-\int_{e}\left(\frac{\partial N}{\partial x}\right)\left(\frac{\partial N}{\partial y}\right)^{T} d \Omega, \quad K 12=(K 21)^{T}, \\
K 31 & =-\int_{e}\left(\frac{\partial N}{\partial x}\right)\left(\frac{\partial N}{\partial z}\right)^{T} d \Omega, \quad K 13=(K 31)^{T}, \\
K 32 & =-\int_{e}\left(\frac{\partial N}{\partial y}\right)\left(\frac{\partial N}{\partial z}\right)^{T} d \Omega, \quad K 23=(K 32)^{T} .
\end{aligned}
$$




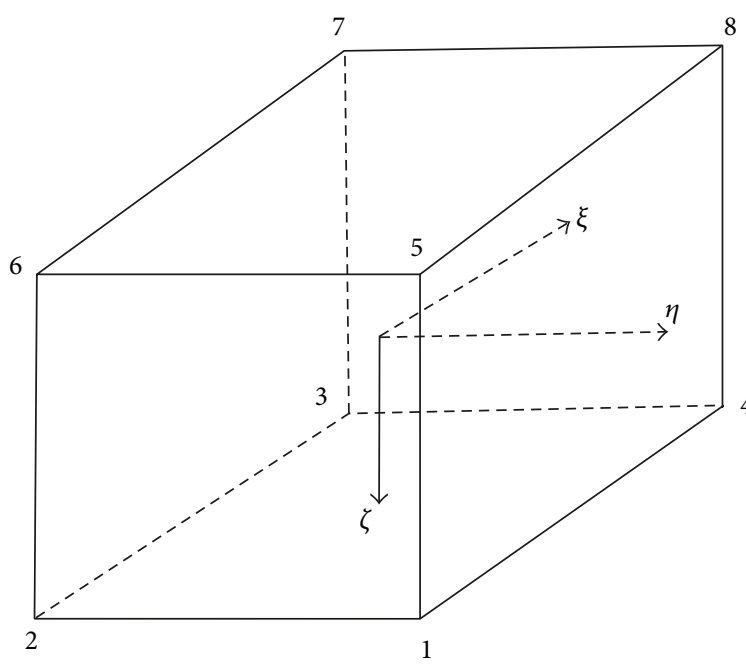

(a)

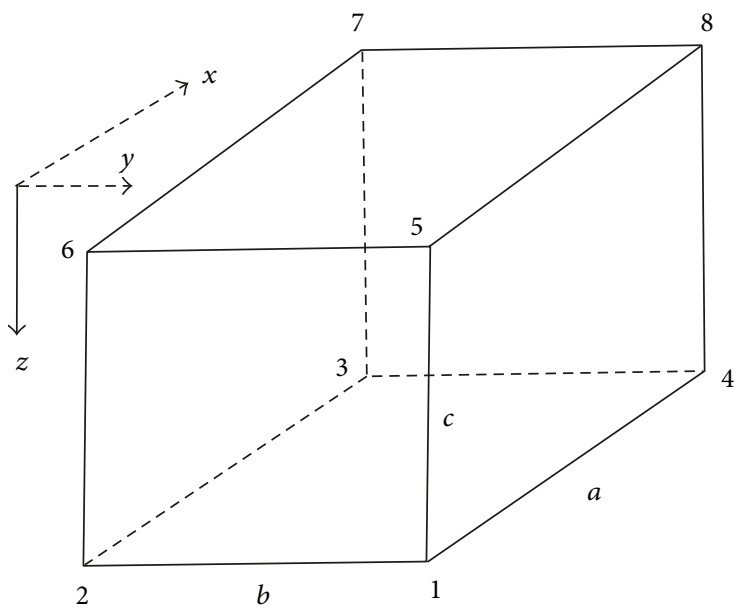

(b)

Figure 4: (a) The parent unit of hexahedron; (b) The subelement of hexahedron.

The expressions of the elements in each matrix are listed where

as

$$
\begin{aligned}
& K 11_{i j}=\int_{e}\left(\frac{\partial N_{i}}{\partial \eta} \frac{\partial N_{j}}{\partial \eta} \frac{4}{b^{2}}+\frac{\partial N_{i}}{\partial \zeta} \frac{\partial N_{j}}{\partial \zeta} \frac{4}{c^{2}}\right) \frac{a b c}{8} d \xi \eta \zeta \\
& =\frac{1}{36}\left(\frac{a c}{b} \beta_{i j}+\frac{a b}{c} v_{i j}\right) \text {, } \\
& K 22_{i j}=\int_{e}\left(\frac{\partial N_{i}}{\partial \xi} \frac{\partial N_{j}}{\partial \xi} \frac{4}{a^{2}}+\frac{\partial N_{i}}{\partial \zeta} \frac{\partial N_{j}}{\partial \zeta} \frac{4}{c^{2}}\right) \frac{a b c}{8} d \xi \eta \zeta \\
& =\frac{1}{36}\left(\frac{b c}{a} \alpha_{i j}+\frac{a b}{c} v_{i j}\right) \text {, } \\
& K 33_{i j}=\int_{e}\left(\frac{\partial N_{i}}{\partial \xi} \frac{\partial N_{j}}{\partial \xi} \frac{4}{a^{2}}+\frac{\partial N_{i}}{\partial \eta} \frac{\partial N_{j}}{\partial \eta} \frac{4}{b^{2}}\right) \frac{a b c}{8} d \xi \eta \zeta \\
& =\frac{1}{36}\left(\frac{b c}{a} \alpha_{i j}+\frac{a c}{b} \beta_{i j}\right) \text {, } \\
& K 21_{i j}=-\int_{e} \frac{\partial N_{i}}{\partial \xi} \frac{\partial N_{j}}{\partial \eta} \frac{c}{2} d \xi \eta \zeta \\
& =-\frac{c}{24} m_{i j} \text {, } \\
& K 31_{i j}=-\int_{e} \frac{\partial N_{i}}{\partial \xi} \frac{\partial N_{j}}{\partial \zeta} \frac{b}{2} d \xi \eta \zeta \\
& =-\frac{b}{24} n_{i j} \\
& K 32_{i j}=-\int_{e} \frac{\partial N_{i}}{\partial \eta} \frac{\partial N_{j}}{\partial \zeta} \frac{a}{2} d \xi \eta \zeta \\
& =-\frac{a}{24} l_{i j}
\end{aligned}
$$

$$
\beta_{i j}=\left|\begin{array}{cccccccc}
4 & 2 & -2 & -4 & 2 & 1 & -1 & -2 \\
& 4 & -4 & -2 & 1 & 2 & -2 & -1 \\
& & 4 & 2 & -1 & -2 & 2 & 1 \\
& & & 4 & -2 & -1 & 1 & 2 \\
& & & & 4 & 2 & -2 & -4 \\
& & & & & 4 & -4 & -2 \\
& & & & & 4 & 2 \\
& & & & & & 4
\end{array}\right| \text {, }
$$

$$
\gamma_{i j}=\left|\begin{array}{cccccccc}
4 & -4 & -2 & 2 & 2 & -2 & -1 & 1 \\
& 4 & -4 & -2 & 2 & 2 & -2 & -1 \\
& 4 & -4 & -2 & 2 & 2 & -2 \\
& & 4 & -4 & -2 & 2 & 2 \\
& & & 4 & -4 & -2 & 2 \\
& & & & 4 & 2 & -2 \\
& & & & & 4 & -4 \\
& & & & & & 4
\end{array}\right| \text {, }
$$

$$
m_{i j}=\left|\begin{array}{cccccccc}
2 & 1 & -1 & -2 & 2 & 1 & -1 & -2 \\
1 & 2 & -2 & -1 & 1 & 2 & -2 & -1 \\
1 & 2 & -2 & -1 & 1 & 2 & -2 & -1 \\
2 & 1 & -1 & -2 & 2 & 1 & -1 & -2 \\
-2 & -1 & 1 & 2 & -2 & -1 & 1 & 2 \\
-1 & -2 & 2 & 1 & -1 & -2 & 2 & 1 \\
-1 & -2 & 2 & 1 & -1 & -2 & 2 & 1 \\
-2 & -1 & 1 & 2 & -2 & -1 & 1 & 2
\end{array}\right| \text {, }
$$




$$
\begin{aligned}
n_{i j} & =\left|\begin{array}{cccccccc}
2 & -2 & -1 & 1 & 2 & -2 & -1 & 1 \\
2 & -2 & -1 & 1 & 2 & -2 & -1 & 1 \\
1 & -1 & -2 & 2 & 1 & -1 & -2 & 2 \\
1 & -1 & -2 & 2 & 1 & -1 & -2 & 2 \\
-2 & 2 & 1 & -1 & -2 & 2 & 1 & -1 \\
-2 & 2 & 1 & -1 & -2 & 2 & 1 & -1 \\
-1 & 1 & 2 & -2 & -1 & 1 & 2 & -2 \\
-1 & 1 & 2 & -2 & -1 & 1 & 2 & -2
\end{array}\right|, \\
l_{i j} & =\left|\begin{array}{cccccccc}
2 & -2 & -2 & 2 & 1 & -1 & -1 & 1 \\
2 & -2 & -2 & 2 & 1 & -1 & -1 & 1 \\
-2 & 2 & 2 & -2 & -1 & 1 & 1 & -1 \\
-2 & 2 & 2 & -2 & -1 & 1 & 1 & -1 \\
1 & -1 & -1 & 1 & 2 & -2 & -2 & 2 \\
1 & -1 & -1 & 1 & 2 & -2 & -2 & 2 \\
-1 & 1 & 1 & -1 & -2 & 2 & 2 & -2 \\
-1 & 1 & 1 & -1 & -2 & 2 & 2 & -2
\end{array}\right| .
\end{aligned}
$$

Substituting (18) into the second integral of (19):

$$
\int_{e} k^{2} E \cdot \delta E d \Omega=\delta E_{e}^{T} K_{2 e} E_{e}
$$

where

$$
\begin{aligned}
& K_{2 e}=\left[\begin{array}{ccc}
K 11 & 0 & 0 \\
0 & K 22 & 0 \\
0 & 0 & K 33
\end{array}\right], \\
& K 11=K 22=K 33=\int_{e} k^{2} N N^{T} d \Omega, \\
& K 11_{i j}=\int_{e} k^{2} N_{i} N_{j} \frac{a b c}{8} d \xi d \eta d \zeta, \quad i, j=1, \ldots, 8 \\
& =\frac{1}{144} a b c k^{2} o_{i j} \\
& o_{i j}=\left|\begin{array}{llllllll}
6 & 3 & 3 & 6 & 2 & 1 & 1 & 2 \\
& 6 & 6 & 3 & 1 & 2 & 2 & 1 \\
& & 6 & 3 & 1 & 2 & 2 & 1 \\
& & & 6 & 2 & 1 & 1 & 2 \\
& & & & 6 & 3 & 3 & 6 \\
& & & & & 6 & 6 & 3 \\
& & & & & & 6 & 3 \\
& & & & & & & 6
\end{array}\right| .
\end{aligned}
$$

When the boundary surface unit 1234 is in the boundary surface EFGH, the boundary integral is

$$
\int_{1234} \chi E_{x} \delta E_{z} d \Gamma=\delta E_{e}^{T} K_{3 e} E_{e}
$$

where

$$
\begin{gathered}
K_{3 e}=\left[\begin{array}{ccc}
K 11 & 0 & 0 \\
0 & 0 & 0 \\
0 & 0 & 0
\end{array}\right], \\
K 11=\int_{1234} \chi N N^{T} d \Gamma, \\
K 11_{i j}=\int_{1234} \chi N_{i} N_{j} \frac{a b}{4} d \xi d \eta, \quad i, j=1, \ldots, 4 \\
=\frac{1}{36} a b \chi p_{i j}, \\
p_{i j}=\left|\begin{array}{rrr}
4 & 1 & 2 \\
4 & 2 & 1 \\
4 & 2 \\
& & 4
\end{array}\right|,
\end{gathered}
$$

where $N_{i}, N_{j}$ are the shape functions of quadrilateral plane element rather than the shape functions of hexahedral plane element. Its form is shown in the following:

$$
N_{i}=\frac{1}{4}\left(1+\xi_{i} \xi\right)\left(1+\eta_{i} \eta\right)
$$

Substituting (14) into (22) and (27), (31) into (29), respectively, each element of the matrix can then be calculated.

\section{The Integration of the Coefficient Matrix}

Integrating all the hexahedral elements obtained above gives the integration of the coefficient matrix which is shown below:

$$
\begin{aligned}
\sum \partial U_{e}^{T}\left(K_{1 e}-K_{2 e}+K_{3 e}\right) U_{e} & =\sum \partial U_{e}^{T} K_{e} U_{e} \\
& =\partial U^{T} \sum K_{e} U=\partial U^{T} K U=0 .
\end{aligned}
$$

The arbitrary $\partial U^{T}$ is not always zero, which gives the following large-scale equations:

$$
K U=0,
$$

where $K$ is the coefficient matrix and $U$ is the unknown variables.

\section{The Computation of Apparent Resistivity}

The expansions of (2) in the Cartesian coordinate system are presented below:

$$
\begin{aligned}
& H_{x}=\frac{1}{i \omega \mu}\left(\frac{\partial E_{z}}{\partial y}-\frac{\partial E_{y}}{\partial z}\right), \\
& H_{y}=\frac{1}{i \omega \mu}\left(\frac{\partial E_{z}}{\partial z}-\frac{\partial E_{z}}{\partial x}\right), \\
& H_{z}=\frac{1}{i \omega \mu}\left(\frac{\partial E_{y}}{\partial x}-\frac{\partial E_{z}}{\partial y}\right) .
\end{aligned}
$$




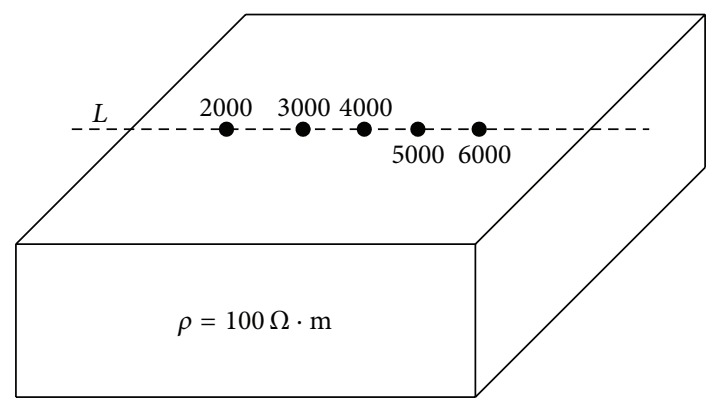

FIGURE 5: Homogeneous model.

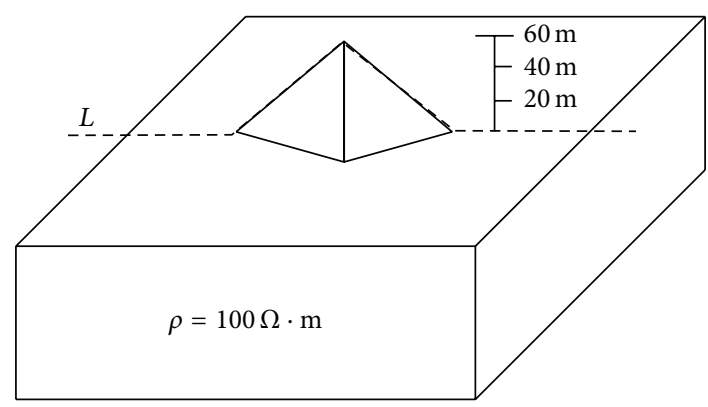

Figure 6: Positive landform model.

When $E_{x}, E_{y}$, and $E_{z}$ of each vertex have been obtained, $H_{x}, H_{y}$, and $H_{z}$ can be computed from the approximate values of each vertex's differential quotient using the difference method. Therefore, the apparent resistivity equation is derived as:

$$
\rho_{x y}=\frac{i}{\omega \mu}\left(\frac{E_{z}}{H y}\right)^{2} .
$$

\section{Example Analysis}

9.1. Homogeneous Model. Figure 5 shows a homogeneous model: the resistivity is $100 \Omega \cdot \mathrm{m}$; the grid size is $9000 \times$ $9000 \mathrm{~m}^{2}$; the dot pitch is $1000 \mathrm{~m}$; the line spacing is $1000 \mathrm{~m}$. The measuring frequency is $1000 \mathrm{~Hz}, 500 \mathrm{~Hz}, 100 \mathrm{~Hz}$, and $50 \mathrm{~Hz}$, and the observation points are the five points of the midline $(2000 \mathrm{~m}, 3000 \mathrm{~m}, 4000 \mathrm{~m}, 5000 \mathrm{~m}$, and $6000 \mathrm{~m})$.

Table 1 lists the numerical computation results. According to the results, the average of the relative error is $2.81 \%$ in $1000 \mathrm{~Hz}$ and $0.16 \%$ in $100 \mathrm{~Hz}$. Gradually decreasing relative error is observed as the frequency decreases.

9.2. Positive Landform Model. In Figure 6, a positive landform model has a resistivity of $100 \Omega \cdot \mathrm{m}$ and the topographic fall is $60 \mathrm{~m}$; the grid size is $9000 \times 9000 \mathrm{~m}^{2}$; the dot pitch is $1000 \mathrm{~m}$; and the line spacing is $1000 \mathrm{~m}$. The measuring frequencies are $1000 \mathrm{~Hz}$ and $100 \mathrm{~Hz}$, and the observation points are the nine points on the midline.

Results of the numerical computation are shown in Figure 7. The highest value is the pseudoabnormality in the positive landform. The distortion error is $39.5 \%$ at $1000 \mathrm{~Hz}$

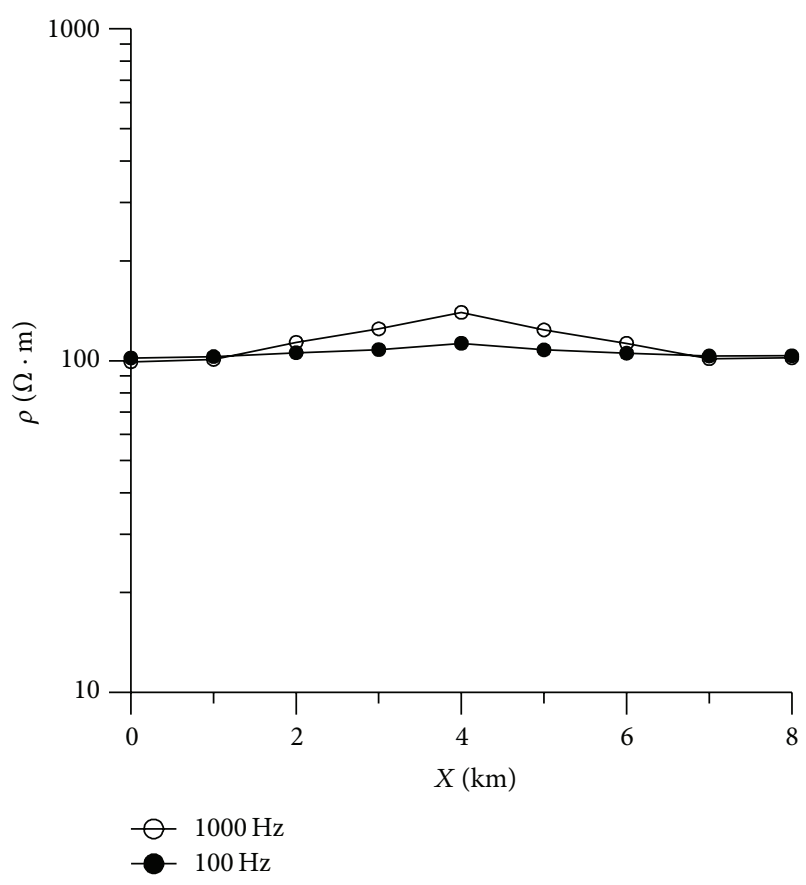

FIGURE 7: The result of numerical computation.

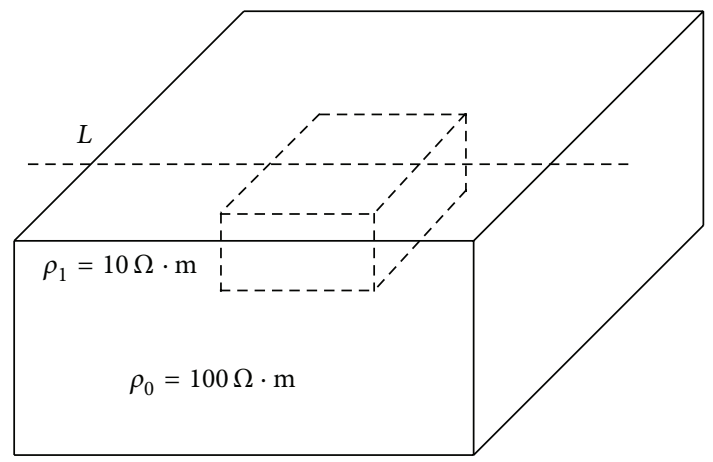

Figure 8: The model with low resistance body.

and $12.8 \%$ at $100 \mathrm{~Hz}$. With a decreasing frequency, the effect of topographic distortion decreases gradually.

9.3. The Model with Low Resistance Body. Figure 8 shows a model containing a low resistance body with $10 \Omega \cdot \mathrm{m}$ resistivity; resistivity of the rest of the model is $100 \Omega \cdot \mathrm{m}$; The horizontal geometry of the anomalous body is $3000 \mathrm{~m} \times$ $3000 \mathrm{~m}$; upper surface is $250 \mathrm{~m}$ away from the ground and its height is $750 \mathrm{~m}$; the grid size is $9000 \times 9000 \mathrm{~m}^{2}$; the dot pitch is $1000 \mathrm{~m}$; the line spacing is $1000 \mathrm{~m}$ (Figure 9). The measuring frequencies are $1000 \mathrm{~Hz}$ and $100 \mathrm{~Hz}$, and the observation points are the nine points of the mid-line.

The result of numerical computation is shown in Figure 10 with the curves showing low resistivity abnormality at both $1000 \mathrm{~Hz}$ and $100 \mathrm{~Hz}$. 


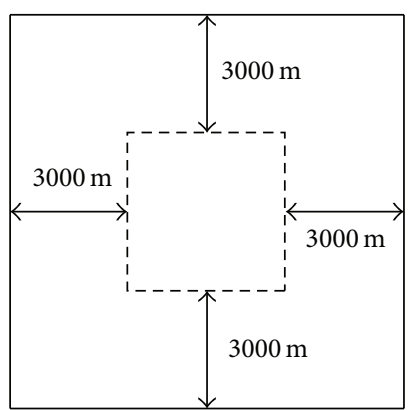

(a)

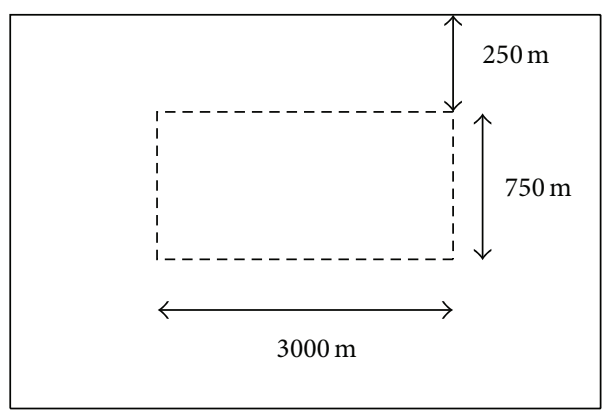

(b)

Figure 9: (a) Horizontal top view. (b) Positive side view.

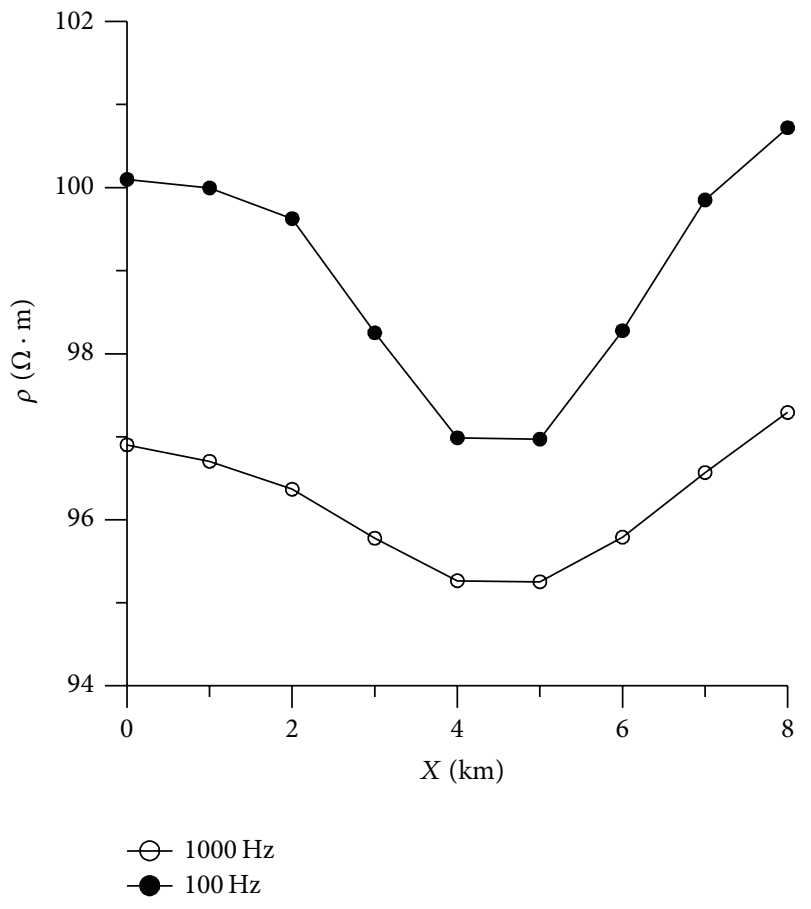

FIgURE 10: The result of numerical computation.

TABLE 1: The result of numerical computation.

\begin{tabular}{lcccc}
\hline$X / \mathrm{Km}$ & Fre/Hz & $\mathrm{Rho} / \Omega \cdot \mathrm{m}$ & Phase $/\left(^{\circ}\right)$ & $\mathrm{RE} /(\%)$ \\
\hline 2000 & 1000 & 97.17 & 44.67 & 2.83 \\
2000 & 500 & 98.26 & 44.66 & 1.74 \\
2000 & 100 & 100.17 & 44.82 & 0.17 \\
2000 & 50 & 99.36 & 45.11 & 0.64 \\
3000 & 1000 & 97.16 & 44.68 & 2.84 \\
3000 & 500 & 98.24 & 44.66 & 1.76 \\
3000 & 100 & 100.14 & 44.83 & 0.14 \\
3000 & 50 & 99.29 & 45.13 & 0.71 \\
4000 & 1000 & 97.17 & 44.68 & 2.83 \\
4000 & 500 & 98.24 & 44.66 & 1.76 \\
4000 & 100 & 100.13 & 44.83 & 0.13 \\
4000 & 50 & 99.23 & 45.14 & 0.77 \\
5000 & 1000 & 97.19 & 44.68 & 2.81 \\
5000 & 500 & 98.26 & 44.66 & 1.74 \\
5000 & 100 & 100.15 & 44.84 & 0.15 \\
5000 & 50 & 99.26 & 45.12 & 0.74 \\
6000 & 1000 & 97.25 & 44.67 & 2.75 \\
6000 & 500 & 98.34 & 44.65 & 1.66 \\
6000 & 100 & 100.21 & 44.85 & 0.21 \\
6000 & 50 & 99.4 & 45.09 & 0.6 \\
\hline
\end{tabular}

\section{Conflict of Interests}

\section{Conclusions and Recommendations}

During the research of magnetotelluric finite element numerical simulation algorithm, (1) the boundary values of 3D magnetotelluric numerical simulations are analyzed; (2) the preconditioned conjugate gradient is adopted for solving large-scale equations under the first-type boundary conditions; (3) the stability and the iterative convergence speed are ensured in 3D magnetotelluric forward calculations. This paper provides an effective tool for theoretical studies on distribution and characteristics of magnetotelluric field response of a 3D geology structure. Meanwhile, it also has established critical foundation for future 3D inversion algorithm research.

The authors declare that there is no conflict of interests regarding the publication of this paper.

\section{Acknowledgments}

The authors would like to acknowledge the great support from the National Key Scientific Instrument and Equipment Development Special Project “3D Frequency Domain Electromagnetic Signal Processing and Inversion Imaging Interpretation System (no. 2011YQ05006007)" and the Key Laboratory of Earth Exploration and Information Technology of Ministry of education in Chengdu University of Technology. 


\section{References}

[1] I. d'Erceville and G. Kunetz, "The effect of a fault on the Earths natural electromagnetic field," Geophysics, vol. 27, pp. 651-665, 1962.

[2] E. Haber, U. M. Ascher, D. A. Aruliah, and D. W. Oldenburg, "Fast simulation of 3D electromagnetic problems using potentials," Journal of Computational Physics, vol. 163, no. 1, pp. 150171, 2000.

[3] R. L. Mackie, T. R. Madden, and P. E. Wannamaker, "Threedimensional magnetotelluric modeling using difference equations-theory and comparisons to integral equation solutions," Geophysics, vol. 58, no. 2, pp. 215-226, 1993.

[4] R. L. Mackie and T. R. Madden, "Conjugate direction relaxation solutions for 3-D magnetotelluric modeling," Geophysics, vol. 58, no. 7, pp. 1052-1057, 1993.

[5] J. T. Smith, "Conservative modeling of 3-D electromagnetic fields, part I: properties and error analysis," Geophysics, vol. 61, no. 5, pp. 1308-1318, 1996.

[6] S. C. Ting and G. W. Hohmann, "Integral equation modeling of three-dimensional magnetotelluric response," Geophysics, vol. 46, no. 2, pp. 182-197, 1981.

[7] P. E. Wannamaker, G. W. Hohmann, and W. A. Sanfilipo, "Electromagnetic modeling of three-dimensional bodies in layered earths using integral equations," Geophysics, vol. 49, no. 1, pp. 60-74, 1984.

[8] Z. Xiong, "Electromagnetic magnetotelluric modeling of 3-D structures by the method of system iteration using integral equations," Geophysics, vol. 57, no. 11, pp. 1556-1561, 1992.

[9] M. S. Zhdanov and V. V. Spichak, Mathematical Modeling of Electromagnetic Fields in Three-Dimensional Inhomogeneous Media, Nauka Publishing House, Moscow, Russia, 1992.

[10] X. Shizhe, The Infinite Element Methods in Geophysics, Science Press, Beijing, China, 1994.

[11] X. Shizhe, R. Baiyao, Zhouhui et al., "3-D terrain effects on magnetotelluric (MT) numerical simulations," Science in China D, vol. 27, no. 1, pp. 15-20, 1997.

[12] C. Xiaobin, Z. Guoze, and M. Xiao, "Research on inversion of MT 3-D modal approximately by 1-D, 2-D inversion method," Chinese Journal of Engneering Geophysics, vol. 3, no. 1, pp. 9-15, 2006.

[13] H. Zuzhi and H. Xiangyun, "Review of three-dimensional magnetotelluric inversion methods," Progress in Geophysics, vol. 20, no. 1, pp. 214-220, 2005.

[14] B. Ruan, S. Xu, and Z. Xu, "Modeling the 3D terrain effect on MT by the boundary element method," Earth Science-Journal of China University of Geosciences, vol. 32, no. 1, pp. 130-134, 2007.

[15] T. Handong, T. Tuo, L. Changhong et al., "Magnetotelluric three-Dimensional modeling using the staggered-grid finite difference method," Chinese Journal of Geophysics, vol. 46, no. 5, pp. 705-711, 2006.

[16] Y. Shu and M. Chen, "Finite element solution of 3-D geo-electric models in frequency magnetotelluric sounding excited by a horizontal electric dipole," Coal Geology \& Exploration, vol. 28, no. 3, pp. 50-56, 2000.

[17] Y. Mitsuhata and T. Uchida, "3D magnetotelluric modeling using the T- $\Omega$ finite-element method," Geophysics, vol. 69, no. 1, pp. 108-119, 2004.

[18] H. Linping and D. Shikun, "Finite element calculation method of 3-D magnetotelluric field under complex condition," Journal of China University of Geosciences, vol. 27, no. 6, pp. 775-779, 2002.

[19] M. J. Nam, H. J. Kim, Y. Song, T. J. Lee, J. Son, and J. H. Suh, "3D magnetotelluric modelling including surface topography," Geophysical Prospecting, vol. 55, no. 2, pp. 277-287, 2007.

[20] F. I. Zyserman and J. E. Santos, "Parallel finite element algorithm with domain decomposition for three-dimensional magnetotelluric modelling," Journal of Applied Geophysics, vol. 44, no. 4, pp. 337-351, 2000.

[21] R. Yoshimura and N. Oshiman, "Edge-based finite element approach to the simulation of geoelectromagnetic induction in a 3-D sphere," Geophysical Research Letters, vol. 29, no. 3, pp. 1039-1045, 2002.

[22] T. Mogi, "Three-dimensional modeling of magnetotelluric data using finite element method," Journal of Applied Geophysics, vol. 35, no. 2-3, pp. 185-189, 1996.

[23] H. A. van der Vorst, "BI-CGSTAB: a fast and smoothly converging variant of BI-CG for the solution of nonsymmetric linear systems," SIAM Journal on Scientific and Statistical Computing, vol. 13, no. 2, pp. 631-644, 1992.

[24] M. Lifeng, W. Xuben, and G. Yongcai, “The probability tomography results of MT and the improved methods," Geophysical Prospecting for Petroleum, vol. 43, no. 6, pp. 612-616, 2004.

[25] X. Shi, J. Wang, S. Zhang, and X. Hu, "Multiscale genetic algorithm and its application in magnetotelluric sounding data inversion," Chinese Journal of Geophysics, vol. 43, no. 1, pp. 122 $130,2000$.

[26] W. Song, “Three-dimensional Fourier transformation migration method of magnetotelluric data and its application," Journal of the University of Petroleum China, vol. 29, no. 4, pp. 21-25, 2005.

[27] W. Wei, Y. Peng, and C. Liping, "Magneto-electrotelluric full frequency segment migration imaging technique and its application," Small Hydrocarbon Reservisions, vol. 12, no. 2, pp. 25-28, 2007. 


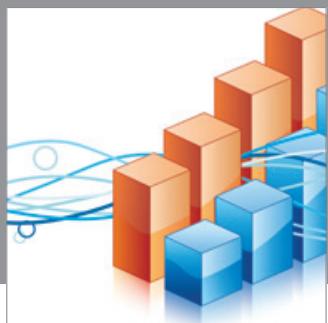

Advances in

Operations Research

mansans

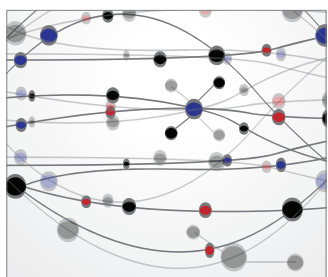

The Scientific World Journal
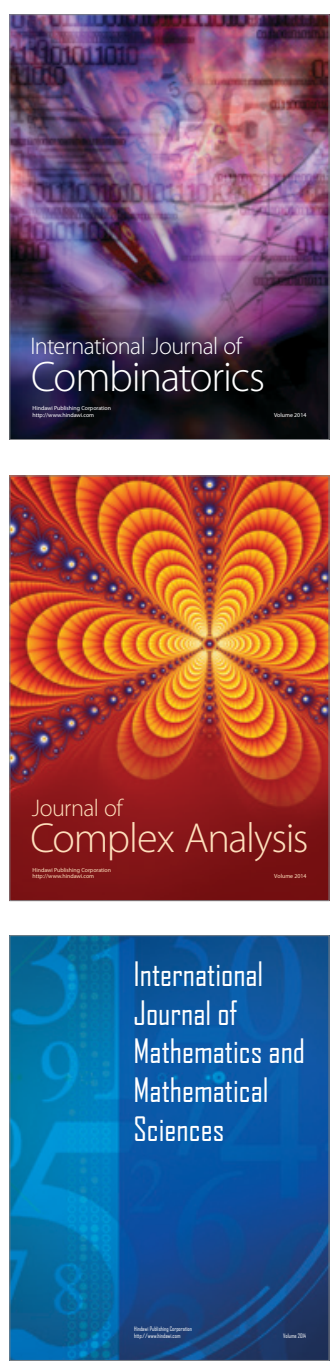
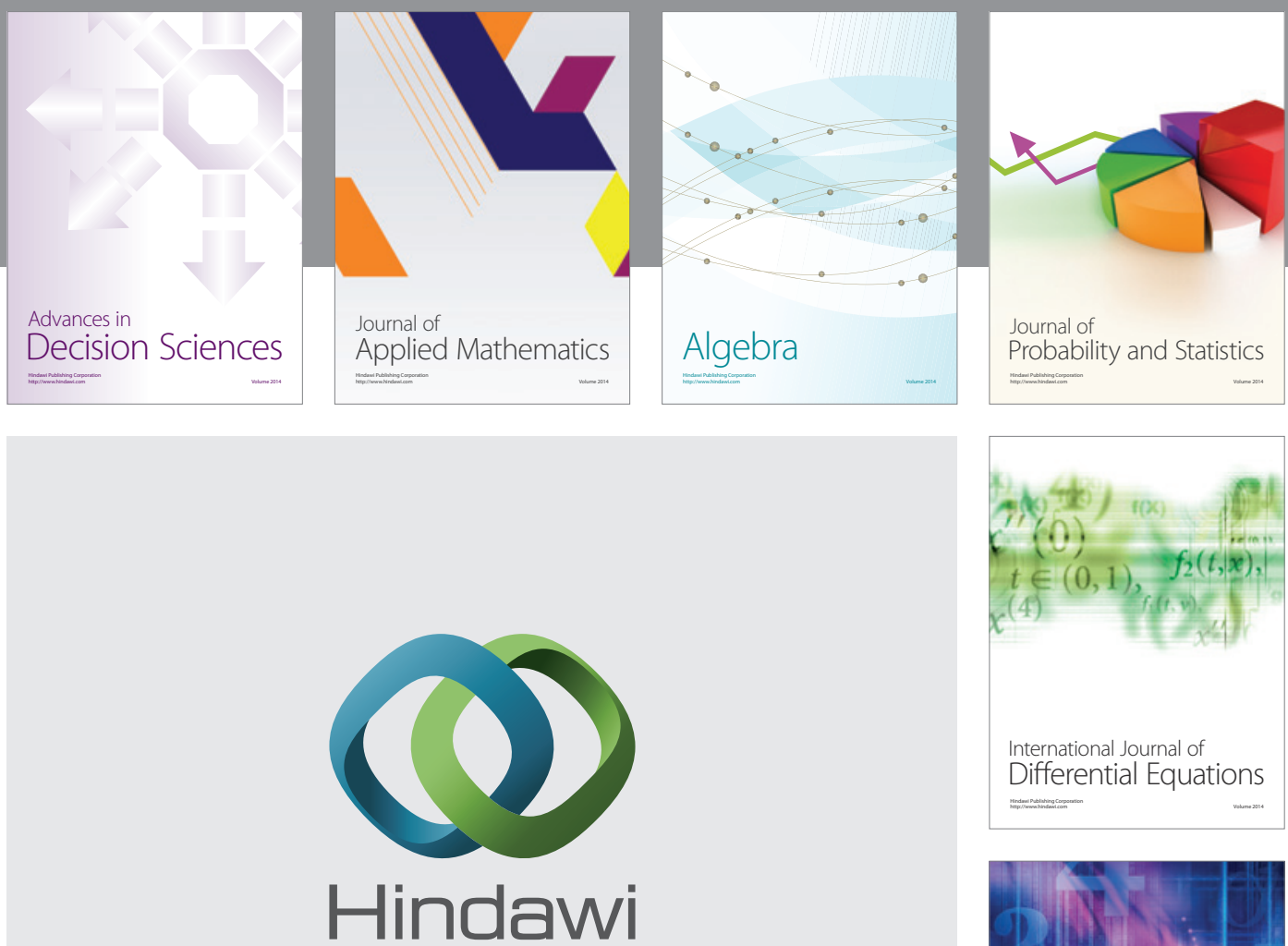

Submit your manuscripts at http://www.hindawi.com
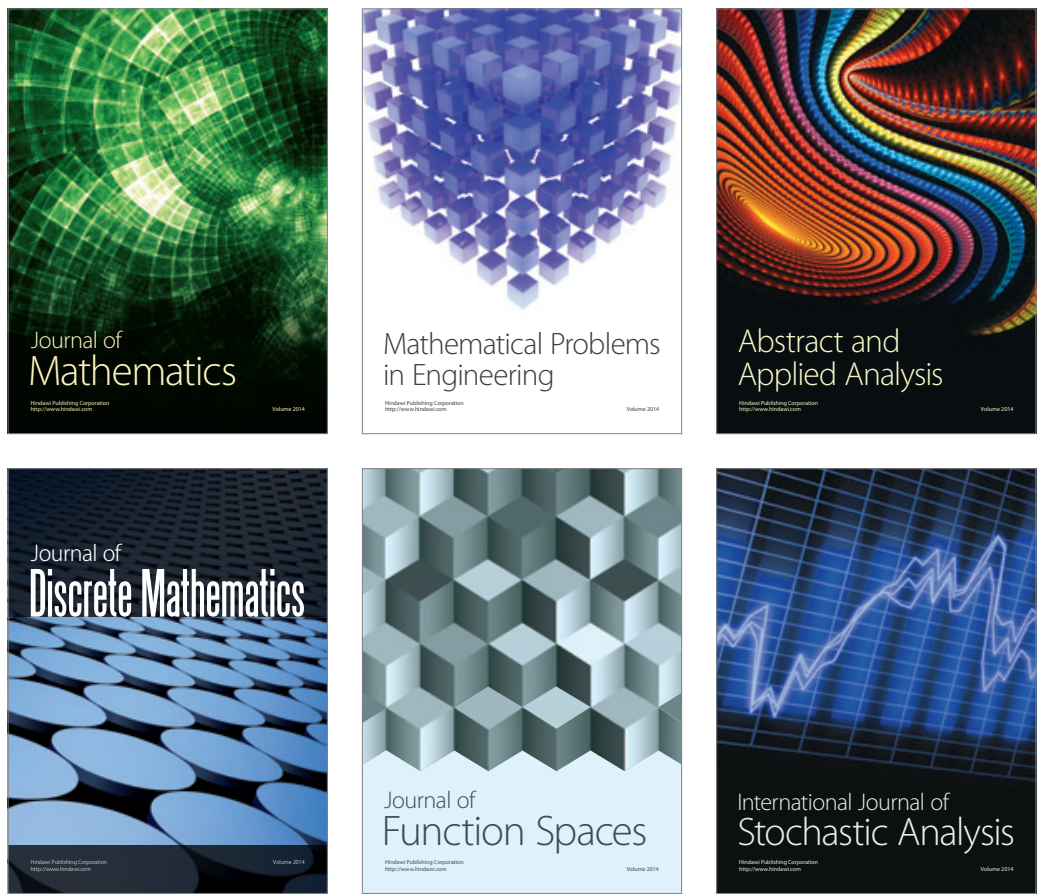

Journal of

Function Spaces

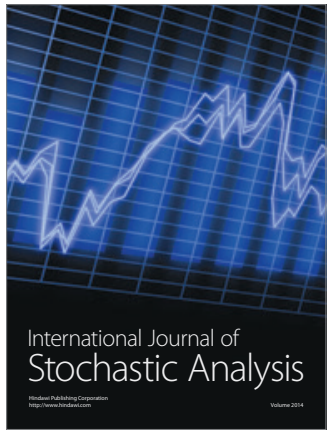

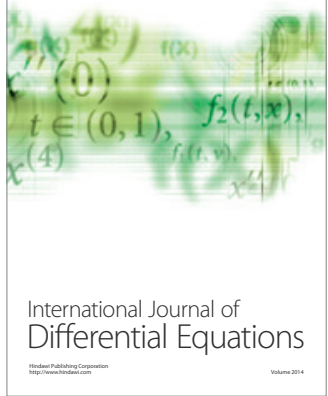
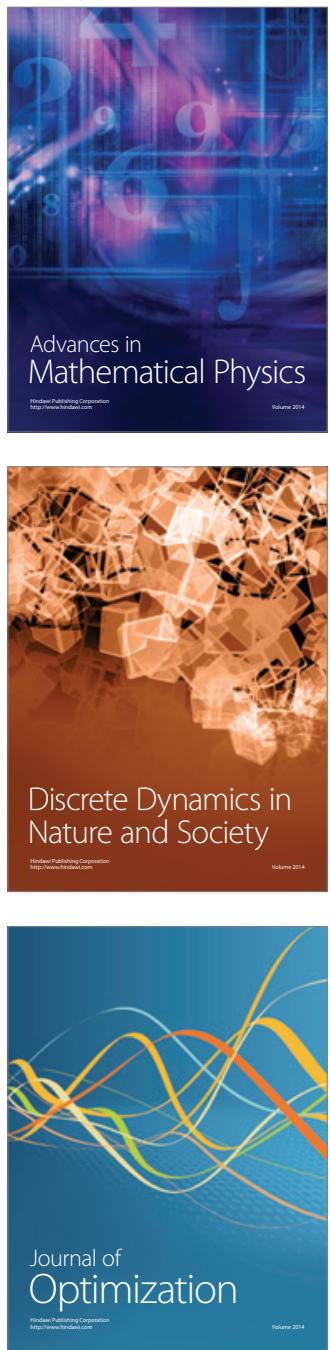